\title{
Editorial
}

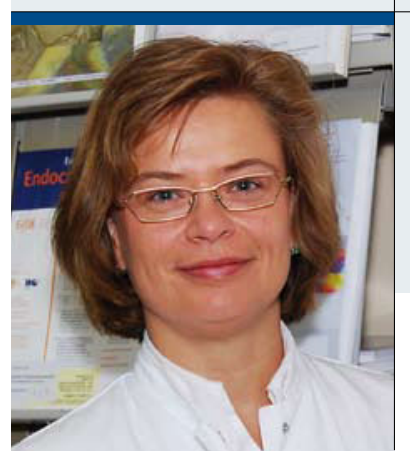

„Wir sollten als Urologen die Chance nutzen, durch die

Weiterbildung in der Andrologie unsere Kompetenz zu stärken,

primärer Ansprechpartner für die Belange des Mannes zu sein."

Prof. Dr. med. Sabine Kliesch

Chefärztin Klinische Andrologie, Centrum für Reproduktionsmedizin

und Andrologie, Münster

\section{Stellenwert der Andrologie wächst}

D ie Andrologie hat in den letzten zehn Jahren nicht nur einen enormen Wissenszuwachs erfahren, sondern auch deutlich an Status in der ärztlichen Weiterbildung gewonnen. Die Zunahme des diagnostischen und therapeutischen andrologischen Wissens lässt der Zusatzweiterbildung Andrologie einen erheblichen Stellenwert im interdisziplinär geprägten Fachgebiet der Männerheilkunde zukommen. Insbesondere bei der Behandlung des infertilen Paares spielt die Andrologie eine wegweisende Rolle. Der Androloge ist integraler Bestandteil eines reproduktionsmedizischen Zentrums, das künstliche Befruchtungen durchführt. Somit spielt die Ejakulatdiagnostik, die zur Basisdiagnostik der Infertilität gehört, eine zentrale Rolle. Um für diesen Laborbereich vergleichbare Qualitätsmaßstäbe zu setzen, werden wir Urologen um die konsequente Durchführung interner und externer Qualitätskontrollen entsprechend den WHO-Richtlinien nicht umhin kommen. Die bislang auf freiwilliger Basis existierende externe Qualitätskontrolle der Deutschen Gesellschaft für Andrologie (QuaDeGA) zeigt sehr deutlich, dass sich durch die regelmäßige Teilnahme an den Ringversuchen und damit verbunden auch an Fortbildungskursen die Qualität in der Diagnostik verbessert.

\section{Kryokonservierung medizinisch und juristisch notwendig}

Wenngleich schon viele Jahrzehnte etabliert, so ist auch die Kryokonservierung von Spermatozoen immer wieder Anlass für Diskussionen. Das Indikationsspektrum reicht von der reinen Fertilitätsprotektion bei onkologischen Patienten über die Samenbanken für die donogene Insemination in entsprechenden Zentren bis hin zur selektiven Kryokonservierung einzelner testikulärer oder epididymaler Spermien bei azoospermen Patienten. Grundsätzlich ist die Kryokonservierung bei allen fertilitätseinschränkenden Maßnahmen des Mannes indiziert - diese Notwendigkeit ist nicht nur eine medizinische, sondern auch eine juristische. Wir haben als Urologen und Andrologen die Pflicht, unsere Patienten, aber auch unsere Kollegen in den benachbarten Disziplinen, über die Möglichkeiten der Kryokonservierung adäquat aufzuklären. Mit den kryokonservierten Proben lassen sich mit den heutigen Verfahren der assistierten Befruchtung auch mittel- und langfristig bei dauerhaft geschädigter Fertilität Kinderwünsche erfüllen.

\section{Ursachen der Infertilität korrekt diagnostizieren}

Darüber hinaus haben in den letzten Jahren vor allem Erkenntnisse der Molekularbiologie und -genetik zu einem deutlich besseren Verständnis der männlichen Infertilität beigetragen. Jedoch ist der diagnostische Spielraum auch in der Früherkennung andrologischer Störungen bei weitem noch nicht ausgeschöpft: Die häufigste numerische Chromosomenaberration in der Urologie und Andrologie, das Klinefelter-Syndrom, wird auch heute nur bei $30 \%$ aller Patienten diagnostiziert - mit teilweise weitreichenden negativen Folgen bezüglich Infertilität und Hypogonadismus. Eine frühe Diagnose könnte Folgeschäden vorbeugen. Bei höhergradigen numerischen Störungen könnte sogar eine positive Beeinflussung der somatischen und psychischen Entwicklung erreicht werden. Beim Subkollektiv der schwerst infertilen Männer mit einer Azoospermie gilt es, vor dem Einsatz eventueller operativer und assistierter Befruchtungsmaßnahmen mögliche genetische Ursachen sorgfältig abzuklären. Diese könnten unerkannt zu Erkrankungen des so sehnlich erwünschten Nachwuchses führen, über die die potenziellen Eltern im Vorfeld zumindest einer umfassenden Aufklärung bedürfen. Bei einer Azoospermie sowie einer schweren Oligozoospermie mit $<10$ Mio. Spermien ist die Indikation zu einer weitergehenden genetischen Diagnostik mit dem Patienten zu besprechen und gleichzeitig eine humangenetische Beratung sinnvoll.

Wir sollten als Urologen die Chance nutzen, durch die Weiterbildung in der Andrologie unsere Kompetenz zu stärken, primärer Ansprechpartner für die Belange des Mannes insbesondere auch bei Fragen der Reproduktion zu sein und unsere Rolle als Partner der Reproduktionsmedizin auszufüllen!

Ihre

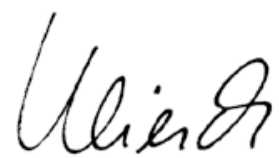

